\title{
Reflets
}

Revue ontaroise d'intervention sociale et communautaire

\section{Barrières linguistiques et intégration des nouveaux immigrants francophones à Toronto}

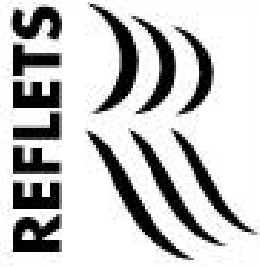

\section{Marie-Claude Muamba}

Volume 4, numéro 1, printemps 1998

Intervention en contextes minoritaires

URI : https://id.erudit.org/iderudit/026205ar

DOI : https://doi.org/10.7202/026205ar

Aller au sommaire du numéro

Éditeur(s)

Reflets : Revue ontaroise d'intervention sociale et communautaire

ISSN

1203-4576 (imprimé)

1712-8498 (numérique)

Découvrir la revue

Citer cet article

Muamba, M.-C. (1998). Barrières linguistiques et intégration des nouveaux immigrants francophones à Toronto. Reflets, 4(1), 182-187.

https://doi.org/10.7202/026205ar

Tous droits réservés (C) Reflets : Revue ontaroise d'intervention sociale et communautaire, 1998
Ce document est protégé par la loi sur le droit d'auteur. L'utilisation des services d'Érudit (y compris la reproduction) est assujettie à sa politique d'utilisation que vous pouvez consulter en ligne.

https://apropos.erudit.org/fr/usagers/politique-dutilisation/ 


\title{
Barrières linguistiques et intégration des nouveaux immigrants franco- phones à Toronto
}

\author{
Marie-Claude Muamba \\ Conseillère à l'emploi, Toronto
}

\section{Introduction}

Cet article est tout simplement une narration et ne se veut pas une étude scientifique. La plupart des éléments mentionnés sont le résultat d'observations et de réflexions personnelles. L'expression «personne immigrante» est utilisée pour désigner aussi bien les immigrants (résidents permanents) que les demandeurs d'asile et les réfugiés au sens de la convention.

Même si le Canada est un pays bilingue, l'usage de l'anglais prédomine. J'ai rencontré beaucoup de personnes venues du Québec pour apprendre l'anglais à Toronto. Dans la plupart des cas, la perte d'un emploi était la raison déterminante de leur déplacement. Cette perte était souvent le résultat du changement survenu dans la définition du poste qu'elles occupaient, qui «d'unilingue français» était devenu bilingue «français-anglais». La problématique de la langue se pose avec acuité pour la personne francophone, immigrante ou non immigrante. Pour la personne immigrante s'installant à Toronto, l'ampleur du problème est double (intégration sociale et emploi-formation). Être bilingue à Toronto est un très grand atout, toutefois l'obtention et le maintien 
d'un emploi pour une personne francophone à Toronto dépendent, non pas de sa connaissance du français, mais surtout de sa maitrise de la langue de Shakespeare. Il y a encore beaucoup de chemin à faire pour que les personnes immigrantes francophones puissent jouir des mêmes privilèges que les anglophones.

\section{Barrières à l'intégration de la personne immigrante francophone à Toronto}

Je vis en Ontario, à Toronto, depuis environ six ans. La diversité des peuples et des communautés y est vraiment frappante. On remarque très rapidement le quartier chinois, le quartier portuguais, Little Italy... Étant francophone, je m'attendais à voir aussi un «Little France». Eh bien, comme vous l'avez probablement deviné, il n'y en a pas.

La diversité de la «communauté francophone» à Toronto mérite d'être mentionnée aussi. Un coup d'oeil rapide permet de constater qu'en dépit de l'usage d'une langue commune, le français en l'occurence, la communauté francophone est aussi hétérogène que la population torontoise. Parmi les francophones, on compte les francophones de souches (de l'Ontario, du Québec, du NouveauBrunswick), ceux d'origines africaine, asiatique, européenne, et antillaise. Cette diversité francophone en elle-même fait que le regroupement dans un quartier est plus difficile à réaliser.

Certes, il existe une communauté francophone à Toronto, il y a des organismes francophones offrant des services en français à Toronto, les enfants peuvent aller dans des écoles francophones. Mais au quotidien, l'anglais est indéniablement présent. Dans l'exercice de mes fonctions de conseillère à l'emploi,je rencontre beaucoup de personnes nouvellement arrivées à Toronto. Je suis tentée de croire que les personnes francophones, d'où qu'elles viennent, ont aussi le désir, comme toutes les autres communautés, d'avoir un espace géographique et social qui, elles l'espèrent, atténuerait les difficultés du processus d'intégration. 
Qu'elle soit voulue ou forcée, il est aussi important de noter que l'immigration entraîne toujours un choc culturel, psycho-social, au contact de la réalité dans le pays d'accueil. Celui-ci revêt l'aspect d'un réveil brutal. C'est la désillusion complète. Un rajustement est nécessaire, il faut réviser ses espérances et ses attentes, ce qui ne se fait pas facilement.

Ajoutons à cela l'isolement auquel font face ces personnes. Plusieurs n'ont pas de famille (restreinte ou élargie) ou d'amis. L'impact du choc varie beaucoup selon la capacité de tout un chacun à gérer la situation et à s'adapter. Parmi les causes responsables du choc, notons les barrières linguistiques, la nonreconnaissance des acquis et de l'expérience acquise ailleurs, l'isolement social, les difficultés financières et morales.

La personne immigrante francophone qui arrive au "Canadapays bilingue» et s'installe en Ontario est malheureusement surprise par le fait que le bilinguisme canadien ne correspond pas à son idée ou à sa notion de bilinguisme. La plupart des immigrants croient qu'au Canada, le français et l'anglais sont utilisés dans la même proportion et invariablement; du moment qu'on parle l'une des deux langues, l'autre n'est pas nécessaire.Je n'ai pas l'intention d'engager un débat sur les langues officielles, néanmoins je pense qu'il est nécessaire d'examiner la question. L'exemple suivant l'illustre amplement. Pour un demandeur d'asile francophone dont la demande a été rejetée, montrer qu'il a appris l'anglais est considéré comme une preuve de son intégration. Est-ce le cas pour la personne anglophone à Toronto? N'a-t-elle pas aussi besoin de prouver son effort d'intégration par l'apprentissage de l'autre langue officielle du Canada? Comme je l'ai déjà dit, la maitrise de l'anglais est indispensable à l'intégration de la personne immigrante.

L'obtention d'un emploi joue aussi un rôle prépondérant dans l'intégration d'une personne dans le pays d'accueil. Une fois l'anglais maîtrisé, la personne immigrante se lance dans la recherche d'un emploi pour découvrir qu'elle n'a pas suffisamment d'expérience canadienne. Les diplômes et compétences acquises en dehors du Canada n'étant pas ou très peu reconnus par les institutions, la personne immigrante se retrouve dans la plupart 
des cas à la croisée des chemins. Faut-il aller à gauche ou à droite? Ou bien rester au centre? S'il y a un centre.

La question fondamentale devient: dois-je retourner aux études ou accepter n'importe quel emploi précaire qui se présentera (circonstances obligent) ou me lamenter sur mon sort et finir par sombrer dans la déprime? C'est ainsi que beaucoup décident de suivre une formation.

Quel programme de formation faut-il suivre? Faut-il se lancer dans une nouvelle branche, un nouveau domaine? Ou faire une mise à jour des connaissances dans son domaine? Aussitôt prise la décision de retourner à l'école, un autre problème se présente: la formation voulue n'est peut-être pas disponible en français, langue que la personne maîtrise et dans laquelle elle peut mieux apprendre. Conséquemment, cette dernière accuse un retard dans la réalisation de ses projets. Il n'y a pas beaucoup d'alternatives: soit qu'elle déménage pour suivre ailleurs la formation en français, soit qu'elle améliore son niveau d'anglais pour pouvoir étudier dans cette langue.

Déménager n'est pas aussi simple que ça. Il y a beaucoup de facteurs à considérer, surtout si on a de la famille. La deuxième alternative serait de suivre ladite formation en anglais, mais la performance aux examens ne sera probablement pas aussi bonne que si les tests étaient passés en français. Il lui faut une bonne dose de persévérance. Une des situations les plus déplorables est celle des gens de métiers. La plupart des métiers sont réglementés en Ontario comme partout ailleurs au Canada. Les personnes immigrants possédant un métier réglementé doivent obtenir un certificat de l'Ontario. Dans certains cas, des heures d'apprentissage chez un employeur et des cours théoriques sont nécessaires à l'obtention de celui-ci. Beaucoup d'immigrants francophones ont dû se décourager et abandonner leurs démarches parce qu'ils n'étaient pas en mesure de trouver un employeur (ni une école) qui fournirait la formation pratique en français.

En ce qui concerne l'emploi, la plupart de mes clients immigrants ont des idées erronnées sur ce qui constitue «un emploi bilingue». La plupart d'entre eux pensent qu'un travail bilingue 
est un travail où l'employé peut, au choix, utiliser la langue qu'il maîtrise le mieux (dans ce cas le français plus que l'anglais). Or ceci n'est pas le cas. Il est souvent nécessaire de questionner les idées préconçues en expliquant qu'un employeur à la recherche d'un employé bilingue veut essentiellement une personne capable d'oeuvrer dans les deux langues officielles du Canada. Les difficultés de ma clientèle en matière de formation et d'emploi sont donc liées mais non limités à des facteurs tels que la maitrise de la langue anglaise, la reconnaissance des acquis et les moyens financiers.

\section{Le service en français}

Parmi les institutions (privées ou publiques) offrant des services bilingues, il y en a qui font des efforts pour offrir un service de qualité. Dans d'autres cas, la qualité ainsi que la rapidité du service ne sont pas garanties. Les intervenants des services communautaires francophones ne disposent pas toujours de tous les outils nécessaires. Il y a des circonstances où les intervenants doivent recourir à des ressources extérieures pour servir le client. Si ces ressources ne sont pas disponibles en français, les intervenants doivent faire appel à un service anglophone. On se bute alors au problème de communication. Le client n'étant pas en mesure de communiquer avec l'intervenant anglophone, il faut faire appel à un service d'interprétation qui n'est pas toujours disponible. Dans ce cas, l'intervenant francophone doit se déplacer ou trouver quelqu'un qui peut et veut aider. Faire appel à une tierce personne ne faisant pas partie du service pose un problème de confidentialité (selon les cas et la nature du problème). Un autre problème peut se poser au niveau de la rémunération de l'interprète. Très peu d'organismes disposent de fonds pour ces services. Souvent le client doit se débrouiller pour en trouver un à ses propres frais, ce qui n'est pas toujours évident. Si l'interprète est bénévole, le service qu'il offre dépend de sa disponibilité. Tout ceci entraîne un retard dans le service fourni au client. 
Un autre facteur concerne l'accessibilité: les organismes offrant les services en français n'étant pas nombreux, les clients doivent souvent se déplacer sur une longue distance pour y accéder. Le service d'emploi du Centre francophone du Toronto Métropolitain reçoit des clients venus d'aussi loin que Oakville, Brampton, Whitby, Ajax. Ceci représente parfois plus d'une heure de voyage ou de transport en commun. Ce facteur joue un rôle prépondérant dans la fréquence d'utilisation du service. Une fois l'anglais maîtrisé, le client ne se sent plus obligé de voyager si loin pour obtenir un service en français alors qu'il peut s'en prévaloir juste à côté en anglais. Dès lors que le client commence à utiliser les services anglophones, la coupure du cordon qui le relie à la communauté francophone n'est plus qu'une question de temps.

Le bilinguisme canadien est, si vous me permettez l'expression, un «bilinguisme du dimanche». Nous n'avons qu'à observer ou plutôt écouter les «employés bilingues" (un échantillon pris au hasard) dans les secteurs publics et privés pour nous en rendre compte. Ma conclusion est la suivante: «anglais excellent, french so so, vous êtes bilingue». 